\title{
INFLUENCE OF FLOW RULE AND INERTIA ON VOID GROWTH IN A RATE SENSITIVE MATERIAL
}

\author{
H. KLÖCKER and F. MONTHEILLET \\ Département Matériaux, École des Mines de Saint-Etienne, 158 \\ Cours Fauriel, F-42023 Saint-Etienne Cedex 2, France
}

Résumé - La déformation d'une cavité ellipsoïdale de révolution dans une matrice infinie soumise à une sollicitation dynamique est étudiée. On montre l'effet stabilisant sur la croissance de $l^{\prime}$ endommagement de la vitesse de traction et des effets $d^{\prime}$ inertie dans le domaine dynamique $\left(\dot{\varepsilon} \geq 10^{3} \mathrm{~s}^{-1}\right)$.

\begin{abstract}
The growth and deformation of a spheroidal void in an infinite viscous matrix is addressed. In the case of a linearly viscous (newtonian) matrix $(\bar{\sigma}=k \dot{\bar{\varepsilon}})$, an exact solution is obtained. On the basis of this solution the case of a power law viscoplastic matrix $(\bar{\sigma}=k \dot{k})$ is dealt with by a variational approach. Finally, the Galerkine method is used to take into account inertia effects. This work shows the anisotropy of damage and the stabilizing effect of the remote strain rate $\dot{\bar{\varepsilon}}$ for a material exhibiting the linear flow rule $\bar{\sigma}=\sigma_{0}+\beta \dot{\bar{\varepsilon}}$ as well as the stabilizing effect of the inertia forces.
\end{abstract}

\section{1. - Introduction}

In mot cases, the ductility of metals and alloys has been shown to increase sharply with strain rate within the dynamic range $\left(\dot{\bar{\varepsilon}}>10^{3} \mathrm{~s}^{-1}\right) / 1 /$. For tensile uniaxial loading, the results can be roughly explained by an enhanced stability of elongation when the strain rate increases. Various mechanical models $/ 2 /$ have shown that the latter phenomenon results from two specific origins of comparable weight occurring in the high strain rate range, namely:

(i) The linear stress-strain rate relationship generally exhibited by the material $/ 3 /$ :

$$
\bar{\sigma}=\sigma_{0}+\dot{\beta \bar{\varepsilon}}
$$

where $\bar{\sigma}$ and $\dot{\bar{\varepsilon}}$ are the von Mises equivalent flow stress and strain rate; the parameters $\sigma_{0}$ and $\beta$ generally depend on strain.

(ii) The inertia effects, which cannot be neglected at high strain rates since very large accelerations are generally involved. Hence, the equation of dynamic equilibrium must be used in the calculations:

$$
\overrightarrow{\operatorname{div}} \sigma=\rho \frac{\mathrm{d} \dot{\overrightarrow{\mathbf{u}}}}{\mathrm{dt}}
$$

Ductile fracture, however, must be considered as the final stage of a continuous damage process involving the initiation, growth and coalescence of microvoids during plastic straining $/ 4 /, / 5 /$. The overall tensile ductility thus does not only depend on the stability 
of the elongation but also on the "intrinsic ductility" (resistance to damage) of the material. Flow rule and inertia effects are expected to affect this damage process as well.

Voids nucleated from severely flattened particles behave with a crack-like character when stressed in particular orientations. This leads to anisotropy in the fracture behaviour. But most of the known void growth models essentially deal with spherical or cylindrical cavities $/ 6 /, / 7 /, / 8 /$. Bilby, Eshelby and Kundu $/ 9 /$ have proposed a model taking into account void geometry, although their method does not give the stress and strain rate distributions within the matrix and is limited to a linearly viscous material $(\bar{\sigma}=\mathbf{k} \overline{\bar{\varepsilon}})$.

In the present paper, the case of a spheroidal void in an infinite homogeneous isotropic matrix under axisymmetric remote loading is addressed (figure 1). The case of a power law viscoplastic matrix $(\bar{\sigma}=\mathrm{k} \dot{\bar{\varepsilon}}$ ) corresponding to quasistatic loading $(\overrightarrow{\operatorname{div}} \sigma=0)$ is studied to bring into evidence the influence of the void excentricity $e=(a-b) /(a+b)$ on its growth rate $\dot{R}$ where $\mathrm{R}=(\mathrm{a}+\mathrm{b}) / 2$. In the case of high speed remote axisymmetric loading, the stabilizing effect of strain rate for a material exhibiting the linear stress-strain rate relationship (1) as well as the stabilizing effect of inertia are then shown.

\section{2. - Stress and strain rate distributions around a spheroidal void within a newtonian matrix}

An exact solution has been obtained in the case of a linearly viscous matrix $/ 10 \%$. This velocity field is the superposition of the homogeneous, remote velocity $\overrightarrow{\vec{u}}$ (without perturbation due to the cavity) and a perturbation term $\dot{\vec{u}}^{*}$ due to the presence of the cavity. The equilibrium equation for a linearly viscous solid may be written in terms of the velocity vector and the pressure gradient $/ 9 /$ :

$$
\Delta \overrightarrow{\mathrm{u}}-\frac{3}{\mathrm{k}} \overrightarrow{\operatorname{grad}} \mathbf{p}=0 \text { and } \operatorname{div} \overrightarrow{\overrightarrow{\mathrm{u}}}=0
$$

The homogeneous velocity field satisfies this equation and the perturbation term will thus be a solution of the same one. Bilby, Eshelby and Kundu /9/ have proposed a general solution to this equation involving a harmonic vector $\vec{B}$ and harmonic scalar $\beta$ :

$$
\overrightarrow{\mathrm{u}}^{*}=\overrightarrow{\mathrm{B}}-\frac{1}{2} \overrightarrow{\operatorname{grad}}(\overrightarrow{\mathrm{r}} \cdot \overrightarrow{\mathrm{B}}+\beta)
$$

In the axisymmetric case this expression reduces to $/ 10 /$ :

$$
\dot{\overrightarrow{\mathrm{u}}}^{*}=\mathrm{B}_{\mathrm{z}} \overrightarrow{\mathrm{e}}_{\mathrm{z}}-\frac{1}{2} \overrightarrow{\mathrm{grad}}\left(\mathrm{z} \cdot \mathrm{B}_{\mathrm{z}}+\beta\right)
$$

where $B_{z}$ and $\beta$ are harmonic potentials in the infinite space outside the void and can be written as a sum of the fundamental harmonics in this space :

$$
B_{z}=\sum_{n=0}^{\infty} a_{n} h_{n}(\vec{r}) \text { and } \beta=\sum_{n=0}^{\infty} b_{n} h_{n}(\vec{r})
$$

where $h_{n}(\vec{r})$ denotes the fundamental harmonics and $a_{n}$ and $b_{n}$ are unknown amplitudes to be determined by the satisfaction of the boundary conditions. Only the harmonics vanishing at infinity must be retained. Then symmetry requirements show that only three fundamental harmonics have to be taken into consideration and the velocity field takes the following form : 


$$
\dot{\overrightarrow{\mathrm{u}}}=\dot{\overrightarrow{\mathrm{u}}}^{\infty}+\dot{\overrightarrow{\mathrm{u}}}^{*}=\dot{\overrightarrow{\mathrm{u}}}^{\infty}+\alpha_{1} \overrightarrow{\mathrm{g}}^{\mathrm{l}}+\alpha_{2} \overrightarrow{\mathrm{g}}^{2}+\alpha_{3} \overrightarrow{\mathrm{g}}^{3}
$$

where $\overrightarrow{\mathrm{g}}^{1}$ corresponds to homothetic void growth without distortion and $\overrightarrow{\mathrm{g}}^{2}$ to pure distortion without volume change. The third fundamental velocity mode $\overrightarrow{\mathrm{g}}^{3}$ contributes to both volume and shape changes of the void and is associated with a non-zero pressure within the matrix. This velocity mode seems to have been neglected in all previous void growth models concerned with power law viscoplastic materials.

The unknown amplitudes $\alpha_{1}, \alpha_{2}$ and $\alpha_{3}$ have been determined by satisfying the boundary conditions on stress (i.e. the stress vector vanishes on the void surface). Imposing to the shear stress $\sigma_{n t}$ to vanish on the void surface implies a zero shear strain rate $\vec{\varepsilon}_{n t}$ and allows the three unknown amplitudes $\alpha_{1}, \alpha_{2}$ and $\alpha_{3}$ to be expressed as functions of the parameters describing the evolution of the cavity, viz. $\dot{R}$ and $\dot{e}$. This method is valid for any non linearly viscous matrix satisfying the normality condition. In the case of a spherical void, the velocity modes, as well as their amplitudes, take a rather simple form in function of the two new variables $\dot{R}$ and $\dot{e} / 11 /$.

\section{3. - Non linearly viscous matrix and inertia effects}

On the basis of the above solution, the quasistatic problem was dealt with by a variational approach $/ 7 /$. The dynamic case was then solved by the Galerkine method. The equilibrium equation $\overrightarrow{\mathrm{div}} \sigma=\rho \mathrm{d \vec {u }} / \mathrm{dt}$ is equivalent to the condition

$$
\int_{V}\left(\overrightarrow{\operatorname{div} \sigma}-\rho \frac{d \dot{\vec{u}}}{d \mathfrak{d}}\right) \cdot \vec{w} d v=0
$$

for any weighting function $\vec{w}$, where the integral is extended over the volume of the matrix. Since two optimization variables, viz. $\dot{R}$ and $\dot{e}$, have to be determined, only two weighting functions $\overrightarrow{\mathrm{w}}^{1}$ and $\overrightarrow{\mathrm{w}}^{2}$ are necessary. The velocity modes $\overrightarrow{\mathrm{g}}^{1}$ and $\overrightarrow{\mathrm{g}}^{2}$ themselves were chosen for that purpose. Thus, in the quasistatic case, the Galerkine method gives the same result as the variational principle.

The quasistatic growth of a spherical void in a power law viscoplastic matrix has been widely investigated in the literature. Here the void elongation during deformation is taken into account. Figure 2 illustrates the very strong dependence of the growth rate (fig. 2a) and the distortion rate (fig. 2b) with respect to the strain rate sensitivity parameter $\mathrm{m}$. For small rate sensitivities the void excentricity e remains very small (i.e. the cavity is rather spherical) and the deformation is less anisotropic as in a linearly viscous matrix. Thus the present work shows the strong dependence of the void growth and distortion rates on void excentricity for any matrix behaviour. It clearly comes out that void elongation may not be neglected in the studies of void growth.

One important feature of the present work was to study the stabilizing effect at a microscopic scale of the remote strain rate $\dot{\varepsilon}$ in the case of a material exhibiting the typical linear stress-strain rate relationship of the dynamic range, $\bar{\sigma}=\sigma_{0}+\beta \dot{\bar{\varepsilon}}$. This effect is significant between $10^{3}$ and $10^{4} \mathrm{~s}^{-1}$. For strain rates smaller than $10^{3} \mathrm{~s}^{-1}$, the matrix behaves like a perfectly plastic material $\left(\vec{\sigma}=\sigma_{\dot{0}}\right)$ and for strain rates higher than $10^{4} \mathrm{~s}^{-1}$ it behaves like a newtonian material $(\bar{\sigma}=\mathrm{k} \dot{\bar{\varepsilon}})$. Figure 3 illustrates the strong decrease of the normalized growth velocity $\dot{R} / \dot{\varepsilon} R$ when the remote strain rate $\dot{\varepsilon}$ varies 
from $10^{3}$ to $10^{4} \mathrm{~s}^{-1}$. The values of the triaxiality parameter $\zeta^{\infty}$ considered correspond to tension tests on notched axisymmetric bars $/ 12 /$.

The equilibrium equation expressed in function of dimensionless variables shows that inertia effects are proportional to the square of the void radius, the square of the remote strain rate and the remote triaxiality. Inertia forces decrease the volume grow th and shape change of the void. Thus the growth and the anisotropy of damage are lowered in the presence of inertia effects (figs $4 a$ and $4 b$ ).

In conclusion, even if it was not its main purpose, the present work has brought into evidence the influence of void elongation on the evolution of damage for any matrix behaviour. In the dynamic range, the most important results are the stabilizing effect of the remote strain rate $\dot{\varepsilon}$ between $10^{3}$ and $10^{4} \mathrm{~s}^{-1}$. In general, this corresponds to a ratio $\sigma_{0} / \beta \dot{\varepsilon}$ between 0.1 and 1 . Inertia effects have a marked stabilizing influence for strain rates greater than $10^{3} \mathrm{~s}^{-1}$ associated with void radii larger than $10 \mu \mathrm{m}$.

\section{REFERENCES}

/1/ GiAnNOTTA J.C., REGAZZONI G. and MONTHEILlET F., J.Phys., Colloque C5, 8 (1985) 49.

12/ DOYON J., KLÖCKER H. and MONTHEILLET F., Int. Conf. on Constitutive Law s for Engineering Materials (ICCLEM), Int. Acad. Pub., Chongqing, China (1989) 359.

13/ HART E.W., Acta Metall., 15 (1967) 351.

14/ MONTHEILLET F. and MOUSSY F., Physique et mécanique de l'endommagement, Les Editions de Physique (1986).

15/ GURLAND J. and PLATEAU J., Trans. Am. Soc. Metals, 56 (1963) 442.

16/ McCLINTOCK F.A., J. Appl. Mech., 35 (1966) 363.

/7/ RICE J.R. and TRACEY D.M., J. Mech. Phys. Solids, 17 (1969) 201.

18/ BUDIANSKY B., HUTCHINSON J.W. and SLUTSKY S., Mechanics of Solids, H. G. Hopkins and M.J. Sewell Eds., Pergamon, (1982) 13.

19/ BILBY B.A., ESHELBY J.D. and KUNDU A.K., Tectonophysics, 28 (1975) 265.

/10/ KLÖCKER H., Analyse théorique de la croissance d'une cavité dans un matériau viscoplastique. Thèse, Ecole des Mines de Saint-Etienne (1991).

111/ KLÖCKER H. and MONTHEILLET F., 6th Int. Conf. on Mechanical Behaviour of Materials (ICM6), Kyoto (1991), to be published.

/12/ BEREMIN F.M., J. Méc. Appl., 4 (1980) 307. 

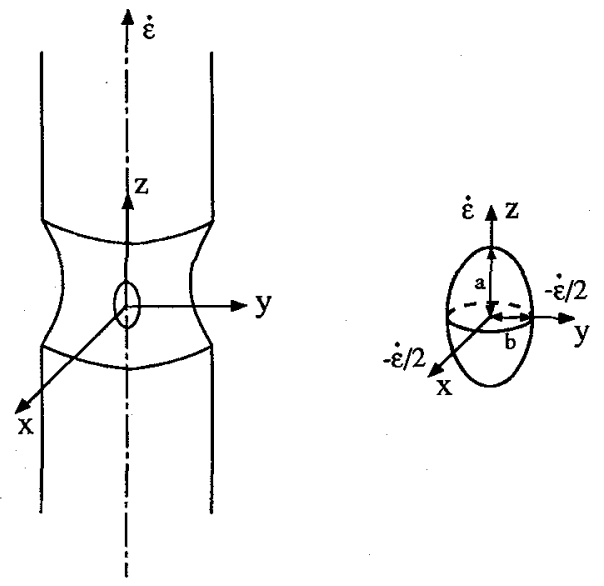

Fig. 1 - Tension test on an axisymmetric specimen.

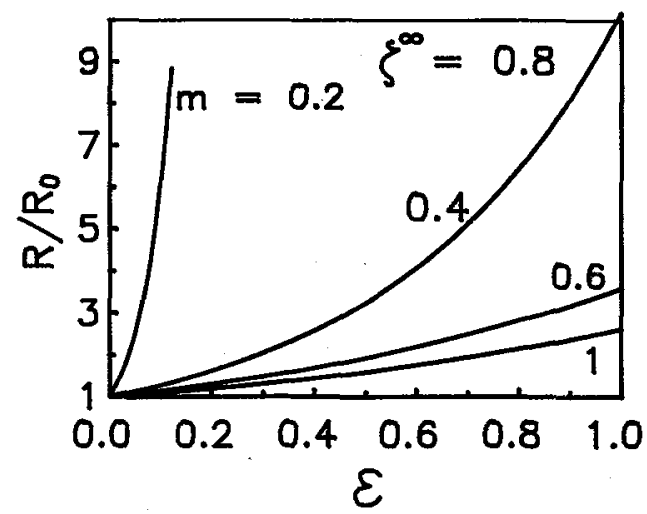

- a -

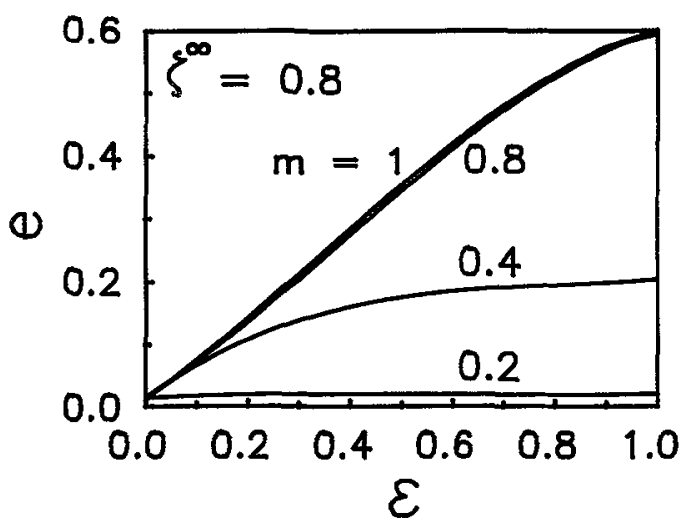

$-b-$

Fig. 2 - Mean void radius $R$ (a) and void excentricity e (b) versus remote strain $\varepsilon$ for a power law viscoplastic infinite matrix. 


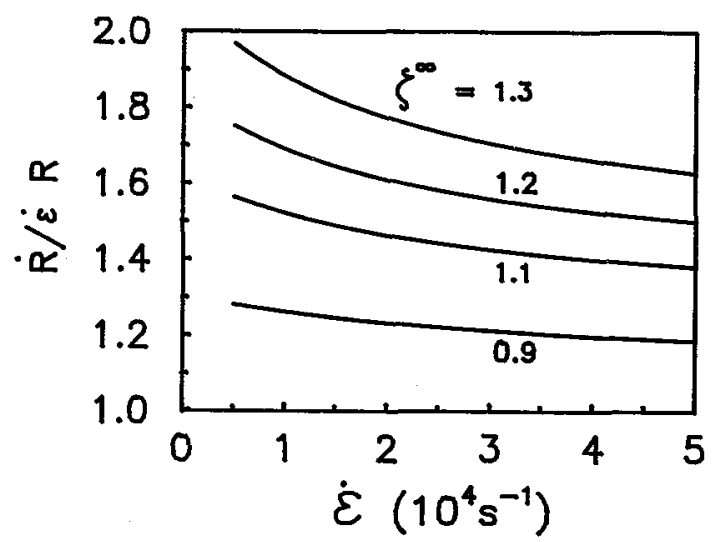

Fig. 3 - Growth rate $\dot{R} / \dot{\varepsilon} R$ versus remote strain rate $\dot{\varepsilon}$ for different values of the triaxiality parameter $\zeta^{\infty}$ corresponding to tension tests on notched specimens.

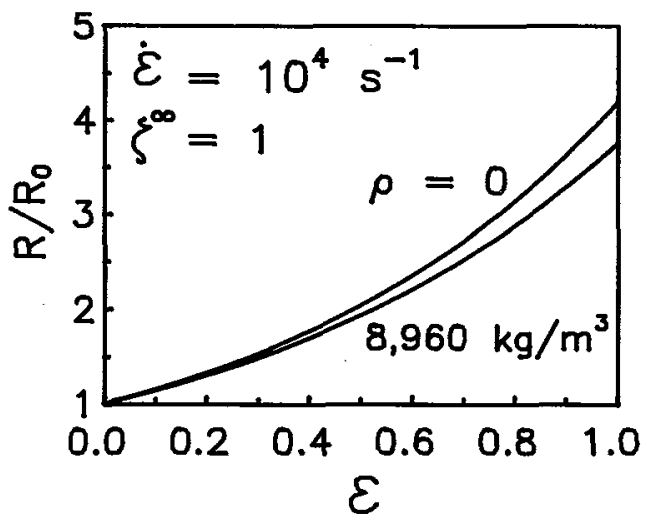

- a -

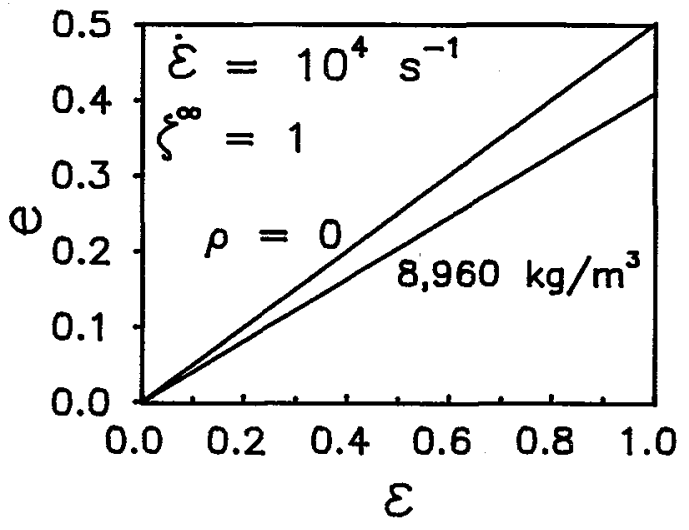

- b -

Fig. 4 - Mean void radius $R$ (a) and void excentricity e (b) versus remote strain $\varepsilon$ in the quasistatic $(\rho=0)$ and the dynamic case $\left(\rho=8,960 \mathrm{~kg} / \mathrm{m}^{3}\right)$. 